\title{
Electronic properties of a quantum wire with arbitrary bending angle
}

\author{
Hua Wu and D. W. L. Sprung \\ Department of Physics and Astronomy, McMaster University, Hamilton, Ontario, L8S 4M1 Canada \\ J. Martorell \\ Dept. d'Estructura i Constituents de la Materia, Facultat Fisica, University of Barcelona, Barcelona \\ 08028, Spain
}

(Received 11 February 1992; accepted for publication 12 March 1992)

\begin{abstract}
The electron transmission and bound state properties of a quantum wire with a sharp bend at arbitrary angle are studied, extending results on the right angle sharp bend (the L-shaped wire). These new results are compared to those of a similar structure, the circular bend wire. The possibility of using a bent wire to perform transistor action is also discussed.
\end{abstract}

\section{INTRODUCTION}

The manifestation of quantal effects in mesoscopic systems is currently a subject of intense interest. ${ }^{1,2}$ Among the many electron wave guide structures which have been studied, bent quantum wires have figured prominently. Sols and Macucci, ${ }^{3}$ Lent, ${ }^{4}$ and ourselves ${ }^{5,6}$ studied the circular bend wire. Exner et al., ${ }^{7}$ Schult et al., ${ }^{8}$ and ourselves ${ }^{6,9}$ have studied wires with right angle sharp bends. An important aim of these studies is to understand electron propagation in these basic structures which could be segments of actual quantum electronic devices. They also serve the purpose of finding efficient methods for numerical modeling of quantum devices. In this paper, we will extend the previous studies by allowing the bending angle to be arbitrary. A special mode matching method is developed to calculate the transmission coefficient and bound state energies. Section II discusses the method, Sec. III gives results on wave propagation, and Sec. IV results for bound states. The conclusions are given in Sec. V.

\section{A SPECIAL MODE MATCHING TECHNIQUE FOR A QUANTUM WIRE BENT AT ARBITRARY ANGLE}

There are many ways to solve the electron waveguide problem: for example, Sols et al. solved the Dyson equation on a tight-binding lattice for the real-space Green's function, ${ }^{10}$ An algorithm based on the boundary element method by Datta et al. ${ }^{11}$ and the quantum transmitting boundary method by Lent $e t$ al. ${ }^{12}$ have also been used. In this paper, however, we will perform a direct mode matching by exploiting the geometry in a novel way. This leads to an algorithm which is simple and fast, as most of the work is done analytically. This is the method of choice when the given geometry is simple enough. $3,5,6,8,9,13-15$

Figure 1 illustrates a quantum wire of width $d$ bent at angle $\pi-\alpha$. As usual, we divide the structure into two leads and a cavity. Due to symmetry, the problem can be solved in half the space with an even and odd solution. We use a mode expansion in the leads

$$
\Psi_{1}=\sum_{n}\left(C_{n} e^{i \alpha_{n} x}+\bar{C}_{n} e^{-i \alpha_{n} x}\right) \phi_{n}(y),
$$

where $\phi_{n}(y)$ is the transverse eigenfunction with energy $\epsilon_{n}$, and

$$
\alpha_{n}=\sqrt{E-\epsilon_{n}}
$$

is the longitudinal wave number associated with the transverse mode $n$. We express energies in units of $\hbar^{2} /\left(2 m^{*}\right)$. The half-cavity of interest here is a right-angle triangle. It is not easy to find a coordinate system conforming to all of its boundaries. This makes a mode expansion of the wave function in the cavity difficult. However, notice that the division of the lead from the cavity is somewhat arbitrary, and the wave function matching boundary is not necessarily a straight line perpendicular to the lead. By relaxing this requirement, we can use a polar coordinate system for the cavity and match the wave function of the lead and the cavity along an arc. For points on this circular arc,

$$
\begin{aligned}
& x=-R(\cos \theta-\cos \alpha / 2), \\
& y=R \sin \theta,
\end{aligned}
$$

where $R=d / \sin (\alpha / 2)$. The wave function mode expansion in the cavity is

$$
\Psi=\sum_{n} f_{n} \sin \left(m_{n} \theta\right) J_{m_{n}}(k r),
$$

where $k=\sqrt{E}$ and

$$
m_{n}=\frac{(2 n-s) \pi}{\alpha}, \quad s=\left\{\begin{array}{lll}
0 & \text { for } & \text { odd symmetry, } \\
1 & \text { for } & \text { even symmetry. }
\end{array}\right.
$$

The matching of wave functions is done by requiring both the wave function and its normal derivative along the arc to be the same when calculated from Eqs. (1) and (5):

$$
\sum_{n} f_{n} \sin \left(m_{n} \theta\right) J_{m_{n}}(k R)=\sum_{n}\left(C_{n} e^{i \alpha_{n} x}+\bar{C}_{n} e^{-i \alpha_{n} x}\right) \sin \frac{n \pi y}{d} .
$$

and

$$
\begin{aligned}
\sum_{n} f_{n} & \sin \left(m_{n} \theta\right) k J_{m_{n}}^{\prime}(k R) \\
= & -\cos \theta \sum_{n} i \alpha_{n}\left(C_{n} e^{i \alpha_{n} x}-\bar{C}_{n} e^{-i \alpha_{n} x}\right) \sin \frac{n \pi y}{d} \\
& +\sin \theta \sum_{n} \frac{n \pi}{d}\left(C_{n} e^{i \alpha_{n} x}+\bar{C}_{n} e^{-i \alpha_{n} x}\right) \cos \frac{n \pi y}{d} .
\end{aligned}
$$




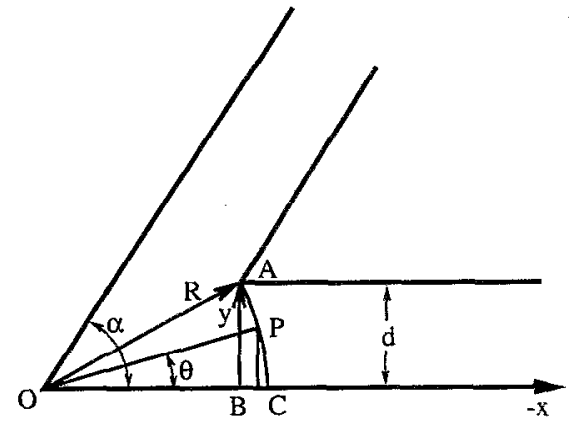

FIG. 1. Quantum wire bent at a sharp angle. $\overline{O A}$ is the symmetry axis, its length is $R=d / \sin (\alpha / 2)$. The lead extends rightward from the line $\overline{A B}$. In region $O A P C B O$, polar coordinates are used, and wave function matching is done along the arc $A P C . P$ is an arbitrary point on the arc.

Multiplying Eqs. (7) and (8) by $\sin m_{p} \theta$ and integrating over $\theta$ from 0 to $\alpha / 2$, we have two sets of linear equations which express $f_{n}$ in terms of $C_{n}$ and $\bar{C}_{n}$. By eliminating $f_{n}$, we obtain a set of linear equations which relate $\bar{C}_{n}$ to $C_{n}$ :

$$
\begin{aligned}
\sum_{n}\left\{\int _ { 0 } ^ { \alpha / 2 } \left[\frac{n \pi}{d} \sin \theta \cos \frac{n \pi y}{d}+\left(i \alpha_{n} \cos \theta-\frac{k J_{m_{p}}^{\prime}(k R)}{J_{m_{p}}(k R)}\right)\right.\right. \\
\left.\left.\quad \times \sin \frac{n \pi y}{d}\right] e^{-i \alpha_{n} x} \sin \left(m_{p} \theta\right) d \theta\right\} \bar{C}_{n} \\
=\sum_{n}\left\{\int _ { 0 } ^ { \alpha / 2 } \left[\left(\frac{k J_{m_{p}}^{\prime}(k R)}{J_{m_{p}}(k R)}+i \alpha_{n} \cos \theta\right) \sin \frac{n \pi y}{d}-\frac{n \pi}{d}\right.\right. \\
\left.\left.\quad \times \sin \theta \cos \frac{n \pi y}{d}\right] e^{i \alpha_{n} x} \sin \left(m_{p} \theta\right) d \theta\right\} C_{n} .
\end{aligned}
$$

Here $x$ and $y$ are functions of $\theta$ as defined by Eqs. (3) and (4). In Eq. (9), $p$ and $n$ are integers: the mode expansion indices with range $(1, \infty)$. The sums are truncated according to the accuracy desired. In Eq. (9), the $C_{n}$ are given input wave function mode amplitudes, and from them, the $\vec{C}_{n}$ are obtained by solving a set of linear equations. Let $\bar{C}_{n \text {,even }}$ and $\bar{C}_{n, \text { odd }}$ be solutions for the even and odd symmetries respectively; then the transmission probability is given by

$$
T=\frac{\Sigma_{n \in \text { open }}\left|\bar{C}_{n, \text { even }}-\bar{C}_{n, \text { odd }}\right|^{2} \alpha_{n}}{4 \Sigma_{n \in \text { open }}\left|C_{n}\right|^{2} \alpha_{n}} .
$$

The homogeneous form of Eq. (9) (i.e., $C_{n}=0$ on the right-hand side) gives the bound states of the system. We compared this method against our previous work on the L-shape wire, ${ }^{9}$ by setting $\alpha=90^{\circ}$, and found excellent agreement. Another test is to calculate the transmission coefficient for $\alpha=\pi$ which is just a straight wire. We expect in this case perfect transmission and no mode mixing. This is a nontrivial test for our method, and we obtain a very accurate result.

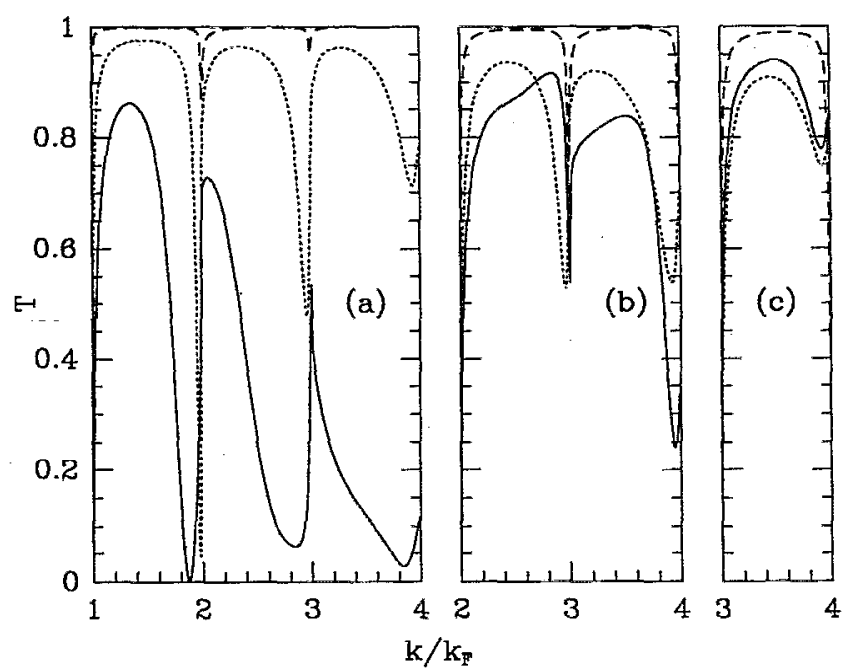

FIG. 2. Total transmission probabilities for a sharp angle quantum wire from the first three input modes, in (a), (b), and (c), respectively. Solid line: $\alpha=90^{\circ}$, dotted line: $\alpha=120^{\circ}$, dash line: $\alpha=150^{\circ}$.

\section{RESULTS FOR TRANSMISSION}

We first discuss results for transmission coefficients. Figure 2 shows the total transmission probabilities for some cases with $\alpha \geqslant 90^{\circ}$. We see a relatively simple structure. As the energy rises, the transmission probability changes smoothly except in a region near the threshold of a new mode ( $k d / \pi$ an integer). This characteristic is similar to the circular bend wire. ${ }^{3}$ The solid lines are for the L-shape wire. As $\alpha$ approaches $\pi$, the devices is more and more close to a straight wire. We expect the transmission probabilities will be close to unity most of the time, as is seen for $\alpha=150^{\circ}$. In fact, the difference between a circular bend and a sharp angle bent wire of very small bending angle, is negligible.

Figure 3 shows the total transmission probabilities for $\alpha<90^{\circ}$. We see that when the bending angle increases, (smaller $\alpha$ ), more and more structure appears in the transmission curves. Roughly speaking, this is due to the increase in area of the cavity, and thus more modes are allowed in the transmitted or reflected waves. When $\alpha \rightarrow 0$, the device region approaches a long rectangle of width $2 d$, while the two leads are parallel and share one common side, a situation discussed by Bar-Touv and Avishai. ${ }^{16}$ While the area of the cavity diverges, it remains closed at the end.

As we have shown in Fig. 3, the total transmission probability is practically unity for very small bending angle. However, as in the case of a circular bend wire, the partial transmission probabilities do vary considerably. In other words, the bent wire converts a wave from one mode to another. Figure 4 is the case of $\alpha=150^{\circ}$ and concerns the first three input modes. Due to symmetry, $T_{i j}=T_{j i}$, where $T_{i j}$ is the transmission probability from mode $i$ to mode $j$. In Fig. 4, the long-dash lines are results for a circular bend of the same bending angle. We can see that they are very close to the sharp angle bend results. The 


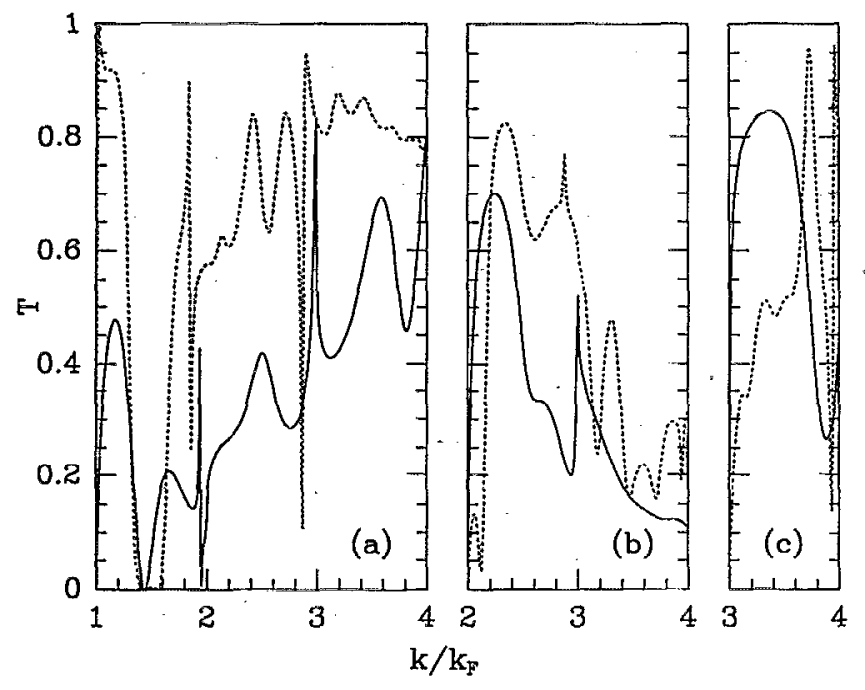

FIG. 3. Total transmission probabilities for a sharp angle quantum wire from the first three input modes, in (a), (b), and (c). Solid line: $\alpha=60^{\circ}$, dotted line: $\alpha=30^{\circ}$.

circular bend results are calculated by a method independent of that in the previous section. Because methods involving Bessel functions have difficulty with the circular bend when the inner radius is zero, we used a method based on a mesh of grid points. Roughly speaking, we used a square mesh in the leads, and in the circular bend region, a grid was placed using polar coordinates. The characteristic mode conversion shown in Fig. 4 is very similar to the circular bend of nonzero inner radius; this can be seen if one compares with Fig. 2(a) of Sols et al. ${ }^{3}$ (note that their $x$ axis is energy while ours is wave number).

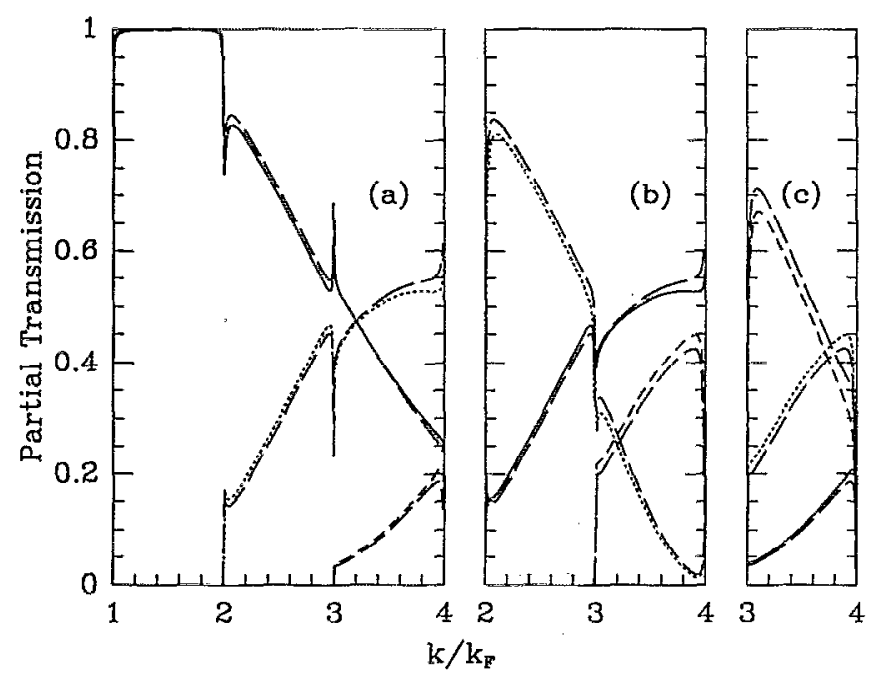

FIG. 4. Partial transmission probabilities $T_{i j}$ for the case $\alpha=150^{\circ}$. Parts (a), (b), (c) for $i=1,2,3$; solid, dotted, and dash lines for $j=1,2,3$. Example: $T_{22}$ corresponds to the dotted line of figure (b). Long dashed lines are the corresponding results for a circular bend with inner radius zero.

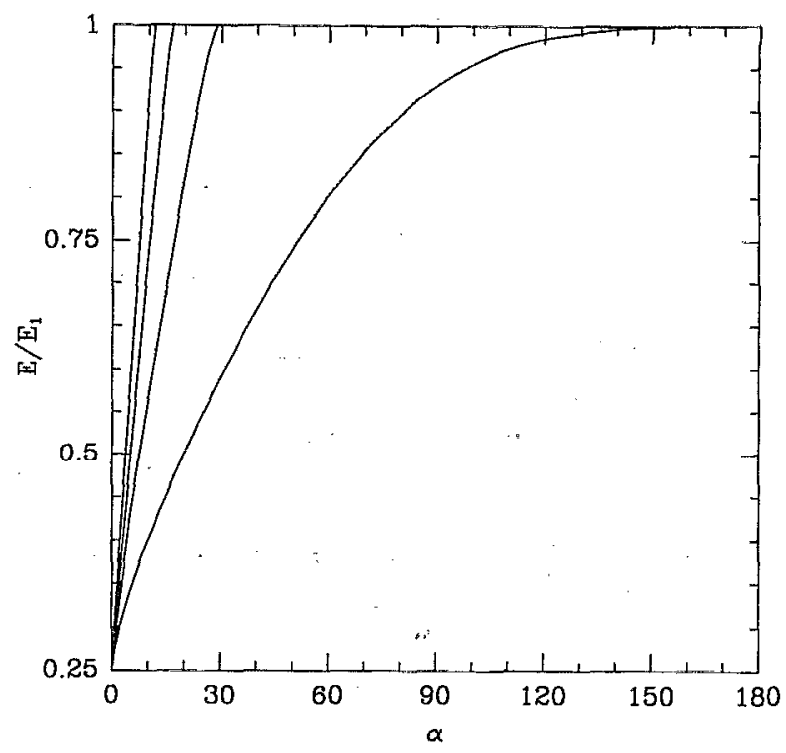

FIG. 5. Bound states of a bent wire as functions of bending angle. The four lines are the energies for the first, second, third, and the fourth eigenstates.

It is interesting to note that transistor action similar to that proposed by $\mathrm{Sols}^{10}$ and Datta $^{2}$ may possibly be achieved with this simple bent wire. As we know, a quantum waveguide structure can be formed by acting with the electrostatic potential of a split gate on a high mobility two-dimensional (2D) electron gas. By changing the applied gate voltage, the effective wire width can be varied. This change is equivalent to a change in the unit of energy $E_{1}$, of the electron propagation in the channel. If the bent wire is operated at a critical value such that a small change in $k d / \pi$ will result in a large change in transmission, then transistor action will be achieved. For example, for a $90^{\circ}$ bend, $k d / \pi=1.8$ is critical (see Fig. 2) and for $\alpha=30^{\circ}$, $k d / \pi=1.2$ is a candidate (see Fig. 3). This type of transistor action is based on quantum interference effects since the region of the bend is acting as a cavity and produces maxima and minima in $T$ similar to those obtained with the cavities proposed by Sols ${ }^{10}$ and Datta. ${ }^{2}$ On the contrary, as is well known, a single pinch on a straight wire works differently, by cutting the number of allowed propagating channels and thus produces a step-function like conductance-gate voltage curve.

\section{RESULTS FOR THE BOUND STATES}

We shall now discuss bound states in a quantum wire bent at an arbitrary angle. In the right angle case, there is only one weakly bound state, symmetric with respect to the two leads at energy $E=0.929 E_{1}$, where $E_{1}$ is the threshold energy for a propagating wave. ${ }^{7-9}$ It is natural to ask the following questions: If the bending angle is reduced, do we still have a bound state and, when the bending angle is larger than $\pi / 2$, could the device have a second bound state? We see in Fig. 5 that when $\alpha$ is not too small, there is a unique bound state. When the bending angle approaches zero $(\alpha \rightarrow \pi)$, the energy goes to $E_{1}$, the threshold 
for a propagating state, and the wave function extends infinitely far into the leads.

The limit of $\alpha \rightarrow 0$ is also quite interesting. In our model with straight edges, the device then becomes a wire of width $2 d$ closed at left infinity, with a wall running from $x=0$ to $+\infty$ down the middle of the wire. The wave function decays very quickly when $x>0$. As an approximation, suppose it vanishes altogether to the right of the origin: $\Psi(x=0, y)=0$. The device then has a continuum of bound states starting at $E=(\pi / 2 d)^{2}=E_{1} / 4$. Of course, for a real wire the part to the left will be of finite area and then only a finite number of states will occur. When $\alpha$ is not zero but small, we can approximate the left segment as a circular sector of radius $2 d / \alpha$ and angle $\alpha$. In other words, we neglect the difference between the arc $A P C$ and line $A B$ in Fig. 1. In this approximation,

$$
\Psi=\sin \left(\frac{\theta n \pi}{\alpha}\right) J_{\pi n / \alpha}(\sqrt{e r}) \quad(0 \leqslant \theta<\alpha) .
$$

The quantization condition requires

$$
J_{\pi n / \alpha}(\sqrt{e} 2 a / \alpha)=0,
$$

and the eigenenergies are then

$$
E / E_{1}=\frac{x_{n i}^{2} \alpha^{2}}{4 \pi^{2}}
$$

where $x_{n i}$ is the $i$ th root of $J_{\pi n / \alpha}(\dot{x})=0$. When $\alpha$ is very small, the order of the Bessel function is very large, and we consider only $n=1$. Equation (13) can be used for small $\alpha$ and provides a simple solution. It is found that the second, third, and fourth bound states appear at $\alpha=28.8^{\circ}, 16.6^{\circ}$, and $11.7^{\circ}$. All these states become degenerate as $\alpha \rightarrow 0$, with energy $0.25 E_{1}$. The bound states shown in Fig. 5 all have even symmetry; no odd symmetry state was found.

\section{CONCLUSION}

The transmission and bound state properties of a sharply bent wire have been studied by a special mode matching method. This is another model for a bent wire, beside the circular bend. For very small bending angle, the results are very similar to a circular bend. It is found that when the bending angle is not so large, there is a unique bound state, but as the bending angle approaches $\pi$, excited bound states do exist. We also discussed the possibility of using a bent wire to perform transistor action.

\section{ACKNOWLEDGMENTS}

We are grateful to NSERC Canada for continuing support under operating grant A-3198 (D.W.L.S and H.W.). The work of J.M. is partially supported by the grant PB87-0311 from DGICYT Spain. D.W.L.S. is also grateful to the Spanish Ministry of Education and Science (DGICYT) for the award of a visiting professorship at the University of Barcelona in the spring term of 1991.

${ }^{\text {I }}$ Special issue on nanoscale devices, Physics Today, 34 (Feb. 1990).

${ }^{2}$ S. Datta, Superlattices and Microstructures 6, 83 (1989).

${ }^{3}$ F. Sols and M. Macucci, Phys. Rev. B 41, 11887 (1990).

${ }^{4}$ C. S. Lent, Appl. Phys. Lett. 56, 2554 (1990).

${ }^{5}$ D. W. Sprung, Hua Wu, and J. Martorell, J. Appl. Phys. 71, 515 (1992).

${ }^{6}$ Hua Wu, D. W. L. Sprung, and J. Martorell, Phys. Rev. B 45, 11960 (1992).

${ }^{7}$ P. Exner, P. Šba, P. Stocicek, Czech. J. Phys. B 39, 1181 (1989).

${ }^{8}$ R. L. Schult, D. G. Ravenhall, and H. W. Wyld, Phys. Rev. B 39, 5476 (1989).

${ }^{9}$ J. Martorell, S. Klarsfeld, D. W. L. Sprung, and Hua Wu, Solid State Commun. 78, 13 (1991).

${ }^{10}$ F. Sols, M. Macucci, U. Ravaioli, and Karl Hess, Appl. Phys. Lett. 54, 350 (1989).

${ }^{11}$ H. Rob Frohne, M. J. McLennan, and S. Datta, J. Appl. Phys. 66, 2699 (1989).

${ }^{12}$ C. S. Lent and D. J. Kirkner, J. Appl. Phys. 67, 6353 (1990).

${ }^{13}$ Y. Avishai and Y. B. Band, Phys. Rev. B 41, 3253 (1990); Y. Avishai, M. Kaveh, and Y. B. Band, ibid. 42, 5867 (1990).

${ }^{14} \mathrm{~K}$.-F. Berggren and Zhen-li Ji, Superlattices and Microstructures 8, 59 (1990).

${ }^{15}$ Hua Wu, D. W. L. Sprung, J. Martorell, and S. Klarsfeld, Phys. Rev. B 44, 6351 (1991).

${ }^{16}$ J. Bar-Tuuv and Y. Avishai, Phys. Rev. B 42, 11496 (1990). 